\title{
Major colorectal resection is feasible using a new robotic surgical platform: the first report of a case series
}

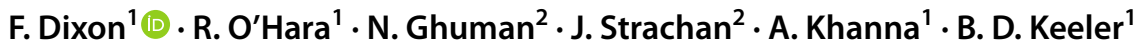

Received: 27 September 2020 / Accepted: 22 October 2020 / Published online: 6 November 2020

(c) Springer Nature Switzerland AG 2020

\begin{abstract}
Background The number of abdominal procedures performed via a robotic-assisted approach is increasing as potential advantages of the modality are recognised. We report the first in human case series of major colorectal resection performed using a new system, Versius ${ }^{\circledR}$, and assess the feasibility of its use.

Methods The initial cases performed using Versius ${ }^{\circledR}$ at a single centre in the UK were included in the study. Anonymised data were prospectively collected including patient demographics, operative details and postoperative outcomes.

Results Twenty-three operations were performed, including left $(n=14)$ and right $(n=9)$-sided colonic resections. Rectal mobilisation was performed in 13. Fifty-seven percent of the patients were male, with a malignant indication for surgery in $70 \%$ of cases. Overall mean age was $59.1 \pm 15.3$ (range 23-89) years. Overall mean body mass index was $28.9 \pm 5.2$ with a mean of $31.3 \pm 4.5$ for left-sided resections. The median console operating time was $166 \mathrm{~min}$ (range 75-320 min). All malignant cases had negative resection margins and the mean lymph node yield was 18 (SD 9.4). Only one operation (4\%) was converted from robotic to open approach. Postoperative length of stay was a median of 5 days (range 3-34 days) and there were no readmissions within 30 days.

Conclusions These results compare favourably with the literature on existing robotic systems and also conventional laparoscopic surgery; hence, we believe that this series indicates the Versius ${ }^{\circledR}$ system is feasible for use in major colorectal resection. These early results from a robot-naïve centre show exciting promise for an expanding robotic market and highlight the need for further evaluation.
\end{abstract}

Keyword Minimally invasive surgery (MIS) $\cdot$ Robotic surgery $\cdot$ Versius $\cdot$ Feasibility $\cdot$ Anterior resection $\cdot$ Right hemicolectomy

\section{Introduction}

Robotic-assisted surgery has been performed for 20 years, with the Food and Drug Administration approval of Intuitive Surgical's da Vinci ${ }^{\circledR}$ robotic system in 2000 . Although initial use was predominantly within urological surgery, there are increasing numbers of procedures performed via

\footnotetext{
B. D. Keeler

Barrie.Keeler@mkuh.nhs.uk

F. Dixon

francesdixon@ doctors.org.uk

1 Department of Surgery, Milton Keynes University Hospital NHS Foundation Trust, Eaglestone, Milton Keynes MK6 5LD, UK

2 Department of Anaesthetics, Milton Keynes University Hospital NHS Foundation Trust, Milton Keynes, UK
}

this approach across multiple specialities prompted by the recognition of the potential advantages of robotic-assisted surgery over open or conventional laparoscopic surgery [1]. These postulated advantages include improved dexterity and stability, particularly within confined spaces such as the pelvis, increasing the ability of a surgeon to perform a procedure via a minimally invasive approach. Such benefits may be particularly advantageous to colorectal surgery with conversion rates from laparoscopic to open approach still in the order of $15 \%[2,3]$.

More widespread uptake of robotic-assisted surgery has potentially been hampered by perceptions of increased costs, size of equipment, and operative setup times [2]. However, current developments in robotic-assisted surgical devices represent an exciting period within surgery. Not only are existing devices undergoing further enhancements, but more platforms are being brought to market. 
The Versius ${ }^{\circledR}$ system from CMR Surgical is one such new platform. It is a modular system with individual arms on bedside units that can be positioned anywhere around the operating table, aimed at providing versatility of port placement for a breadth of surgical procedures whilst also enabling its use in smaller operating theatre footprints. Its design is aimed at overcoming some of the shortcomings of robotic-assisted devices and has been optimised based on feedback from a number of surgeons [4]. Versius ${ }^{\circledR}$ also has an open console design which is intended to improve communication between the operating surgeon and the wider theatre team [5]. We report the first in human series of major colorectal resections performed using Versius ${ }^{\circledR}$ in a single, previously robot-naïve, centre in the UK.

\section{Materials and methods}

Anonymised data were collected on all consecutive major colorectal resections performed with the use of Versius ${ }^{\circledR}$ (CMR Surgical, 1 Evolution Business Park, Cambridge, UK) during an operational robotic-assisted surgical programme of 20 weeks. This excluded two hiatuses to the programme, one during a period of staff annual leave and another due to the impact of COVID-19 on operating practices within the UK. Data were recorded in a prospectively maintained local database as well as a national registry, for which written consent was obtained from every patient. All procedures were performed by two consultant colorectal surgeons. Baseline demographic data were collected along with operation type and indication. Body mass index (BMI) was recorded for all patients. Patient comorbidity status was assessed using the Charlson Age-Comorbidity Index which has been validated for use in a variety of general surgical contexts [6]. American Society of Anaesthesiologists Physical Status Classification System (ASA) was also recorded. Perioperative parameters documented were console operating time (as defined as the total length of time spent performing robotic-assisted dissection), duration of docking of bedside units (BSU) to patient, estimated blood loss intraoperatively, total length of stay, and pain scores on postoperative day 1,2 and 3. Pain was graded on a scale from 1 (no pain) to 10 (severe pain) and recorded by ward nursing staff. Any conversions from robotic-assisted to a different modality were recorded, along with the rationale for this decision.

Postoperative complications were monitored and graded according to the Clavien-Dindo classification [7], and patients continued to be monitored for complications or readmission up until 30 days post-surgery. Complications were classified as major if assigned a Clavien-Dindo grade of III or over. Histological parameters were also collected for all malignant cases, including circumferential resection margin (CRM) and lymph node status.

Results are presented as mean \pm standard deviation for parametric data, and median (range) for non-parametric data.

\section{Results}

Twenty-three consecutive cases were performed during this period. These resections were for both benign (30\%) and malignant indications, and included patients with a wide variety in age (mean $59.1 \pm 15.3$, range $23-89$ years) and comorbid status (Table 1). The mean BMI was $28.9 \pm 5.2 \mathrm{~kg} /$ $\mathrm{m}^{2}$, with a clear majority (74\%) of patients being overweight and 11 being obese (defined as BMI $>30 \mathrm{~kg} / \mathrm{m}^{2}$ ). The majority of patients had at least one significant comorbidity as reflected by a median Charlson Comorbidity Index score of 4 (range 0-9). All patients had an ASA score of $2(87 \%)$ or $3(13 \%)$.

The 23 operations performed included 9 right or extended right hemicolectomies, 1 left hemicolectomy, 6 anterior resections, 4 abdominoperineal excisions of the rectum and a low Hartmann's procedure. There was also one completion proctectomy for ulcerative colitis and one panproctocolectomy for synchronous rectal and caecal cancer. Median operative console time is illustrated in Table 2, along with the duration of bedside unit setup.

Table 1 Patient characteristics

\begin{tabular}{llll}
\hline Characteristics & Right $(n=9)$ & Left $(n=14)$ & Overall $(n=23)$ \\
\hline Sex, male/female & $6 / 3$ & $7 / 7$ & $13 / 10$ \\
Age (years), mean \pm SD & $53.8 \pm 16.0$ & $62.5 \pm 13.8$ & $59.1 \pm 15.3$ \\
ASA score, $n(\%)$ & & & \\
2 & $9(100 \%)$ & $11(79 \%)$ & $20(87 \%)$ \\
3 & $0(0 \%)$ & $3(21 \%)$ & $3(13 \%)$ \\
Charlson comorbidity index, median (range) & $1(0-5)$ & $4(0-9)$ & $4(0-9)$ \\
Body mass index $\left(\mathrm{kg} / \mathrm{m}^{2}\right)$, mean $\pm \mathrm{SD}$ & $25.2 \pm 4.0$ & $31.3 \pm 4.5$ & $28.9 \pm 5.2$ \\
Indication for surgery, malignant/benign & $4 / 5$ & $12 / 2$ & $16 / 7$ \\
Preoperative radiotherapy, $n(\%)$ & $0(0 \%)$ & $3(21 \%)$ & $3(13 \%)$ \\
\hline
\end{tabular}

ASA American Society of Anesthesiologists 
Table 2 Perioperative and postoperative outcomes

\begin{tabular}{lccc}
\hline Outcome & Right $(n=9)$ & Left $(n=14)$ & Total $(n=23)$ \\
\hline Perioperative & & & \\
Console time (minutes), median (range) & $154(75-223)$ & $198(78-320)$ & $166(75-320)$ \\
BSU setup time (minutes), median (range) & $15(11-18)$ & $18(7-39)$ & $17(7-39)$ \\
Conversion to alternative modality, $n(\%)$ & $0(0 \%)$ & $1(7 \%)$ & $1(4 \%)$ \\
Pain score (out of 10), median (range) & & & \\
Day 1 postop & $4.5(0-8)$ & $3(0-7)$ & $4(0-8)$ \\
Day 2 postop & $5(0-9)$ & $4(0-10)$ & $4.5(0-10)$ \\
Day 3 postop & $4(0-8)$ & $3.5(0-7)$ & $4(0-8)$ \\
Length of stay (days), median (range) & $5(3-10)$ & $5.5(4-34)$ & $5(3-34)$ \\
Postoperative & & & $9(39 \%)$ \\
Complication within 30 days, $n(\%)$ & $4(44 \%)$ & $5(36 \%)$ & $7(30 \%)$ \\
$\quad$ Minor, $n$ (\%) & $4(44 \%)$ & $3(21 \%)$ & $2(9 \%)$ \\
$\quad$ Major, $n$ (\%) & $0(0 \%)$ & $2(14 \%)$ & $0(0 \%)$ \\
Readmission within 30 days, $n(\%)$ & $0(0 \%)$ & $0(0 \%)$ & $2(9 \%)$ \\
Reoperation within 30 days, $n(\%)$ & $0(0 \%)$ & $2(14 \%)$ & \\
\hline
\end{tabular}

$B S U$ Bedside unit

Estimated blood loss was less than $100 \mathrm{ml}$ for all operations, except in one patient whose total loss was between 100 and $500 \mathrm{ml}$. The main losses were during the perineal component of an extralevator abdominoperineal excision of rectum (ELAPE). The median length of stay postoperatively was 5 days. This ranged from 3 days for a right hemicolectomy to 34 days for a patient who had a perineal wound infection requiring vacuum dressing therapy. Local community services were unable to manage this dressing type and so the patient remained in hospital for wound management even after being surgically fit for discharge. Pain scores are illustrated in Table 2.

Only one procedure was converted to an open approach. This patient was one of the first cases undertaken, and had a BMI of $33 \mathrm{~kg} / \mathrm{m}^{2}$ with significant diverticular disease-associated adhesions and a technically challenging mobilisation of the splenic flexure. Two further cases were undertaken as a planned hybrid procedure. The first of these was an ELAPE procedure (BMI $36.1 \mathrm{~kg} / \mathrm{m}^{2}$ ) whereby a simultaneous oophorectomy was required due to increased ovarian fluorodeoxyglucose (FDG)-avidity on positron emission tomography (PET) imaging. As this procedure was planned laparoscopically, aspects of the colorectal dissection were also performed by this approach. The second case, panproctocolectomy for synchronous caecal and rectal malignancy, required laparoscopic mobilisation of the splenic flexure to avoid surgeon fatigue for the main oncological components of the procedure.

Significant laparoscopic mobilisation was performed in only two patients. In only one patient was this due to technical difficulty because of post-radiotherapy changes in a male (BMI $33 \mathrm{~kg} / \mathrm{m}^{2}$ ) requiring ELAPE. The other laparoscopic mobilisation was due to subsequent realisation that a further
$1 \mathrm{~cm}$ length of dissection was required after the robot had been undocked.

All malignant cases $(n=16)$ had negative resection margins. Tumour characteristics are described in Table 3. The median number of lymph nodes retrieved, for all samples with a recorded yield (benign and malignant), was 18 (range 7-45). Five patients had positive lymph nodes and were referred for adjuvant chemotherapy. For all rectal cases, the pathologically reported dissection plane was mesorectal fascia.

Nine patients (39\%) had postoperative complications within the first 30 days postoperatively, which are detailed in Table 4. Seven of these complications were minor (Clavien-Dindo grade I or II) and treated pharmacologically alone, but two complications necessitated a return to theatre. One post-radiotherapy patient developed a perineal wound infection post-ELAPE that required washout and examination under anaesthesia, and one patient underwent open

Table 3 Tumour characteristics for malignant cases

\begin{tabular}{lr}
\hline TNM staging & $n=16$ \\
\hline Primary tumour, $n(\%)$ & $5(31 \%)$ \\
T1 & $5(31 \%)$ \\
T2 & $6(38 \%)$ \\
T3 & \\
Regional lymph nodes, $n(\%)$ & $11(69 \%)$ \\
N0 & $5(31 \%)$ \\
N1a & \\
Metastasis, $n(\%)$ & $16(100 \%)$ \\
M0
\end{tabular}


Table 4 Complications by Clavien-Dindo grade

\begin{tabular}{ll}
\hline Grade & Description \\
\hline Grade I & Urinary retention $(n=1)$ \\
Grade II & Wound infection requiring antibiotics $(n=3)$ \\
& Bell's palsy requiring steroids $(n=1)$ \\
& Anastomotic bleed requiring transfusion $(n=1)$ \\
& Cutaneous reaction to skin glue requiring medical therapy $(n=1)$ \\
Grade IIIb & Perineal wound infection requiring examination under anaesthe- \\
& sia and vacuum dressing $(n=1)$ \\
& Extraction site hernia requiring open surgical repair $(n=1)$ \\
\hline
\end{tabular}

surgical repair of an abdominal extraction site hernia. No patients were readmitted within 30 days from discharge.

\section{Discussion}

Previous trials comparing open with laparoscopic colorectal resection have demonstrated conversion rates of between $14.6 \%$ (ALCCaS) and 16\% (COLOR II) from laparoscopy to open $[3,8]$. The rate in this series was $4 \%$ (1 case). Although this initial case series is limited by relatively small numbers, the conversion rate remains comparable to the $8 \%$ rate reported in the ROLARR trial [9].

Risk of conversion to open was significantly higher in the COLOR II trial for patients with BMI of more than $25 \mathrm{~kg}$ / $\mathrm{m}^{2}$, and similarly for those with BMI of more than $30 \mathrm{~kg}$ / $\mathrm{m}^{2}$ in the ROLARR trial $[8,9]$. In this series, nearly threequarters of the patients $(n=17)$ had a BMI of over $25 \mathrm{~kg} / \mathrm{m}^{2}$. All of the patients converted to either open or laparoscopic approaches had BMI of $33 \mathrm{~kg} / \mathrm{m}^{2}$ or higher.

The overall median length of stay was 5 days, with patients with right-sided resections staying 5 days and those with left sided resections staying 5.5 days. This is in keeping with studies comparing laparoscopic and robotic right hemicolectomies [10] and is lower than the ROLARR trial which recorded a median of 8 days stay for rectal resections [9].

None of the 16 malignant cases reported in the present study had a positive resection margin (CRM). This result is reflected in a recent meta-analysis which compared open, laparoscopic and robotic right hemicolectomies, and reported a $0 \%$ positive resection margin rate for the robotic cases [10]. This series compares favourably with the ROLARR trial, which reported a positive CRM in $5.7 \%$ of the cases in their robotic-assisted rectal resection group [9].

The rate of complications within 30 days was $39 \%$ in this series. This has been reported at between 10 and $32 \%$ in a meta-analysis, and $33.1 \%$ in the ROLARR trial $[9,10]$. We are continuing to monitor this patient group up to 90 days post-discharge to ensure no delayed complications become evident.
The console operating time varied widely within this series which reflects the breadth of procedures performed, and the challenging nature of the patient cohort, with high average BMI. This institution was previously robot-naive and we therefore anticipate a reduction in operative procedural durations as the learning curves of both the surgeons and the institution are overcome.

\section{Conclusions}

These results demonstrate that the Versius ${ }^{\circledR}$ system appears feasible for use in major colorectal resectional surgery for both benign and malignant disease, with apparent safe and acceptable outcomes. This includes patients with a high BMI. These early results from a robot-naive centre show exciting promise for an expanding robotic market and highlight the need for further evaluation.

Author contributions All authors meet the ICMJE authorship criteria. All authors contributed to the study conception and design. Material preparation, data collection and analysis were performed by FD and BK. The first draft of the manuscript was written by FD and all authors commented on subsequent versions of the manuscript. All authors read and approved the final manuscript.

Funding No funding was received for this work from any funding agencies in the public, commercial or not-for-profit sectors.

Data availability The datasets generated during and/or analysed during the current study are available from the corresponding author on reasonable request.

Code availability Not applicable.

\section{Compliance with ethical standards}

Conflict of interest The authors declare that they have no real or potential conflicts of interest.

Ethical approval This research was performed in accordance with the ethical standards of the institutional and/or national research committee 
and with the 1964 Declaration of Helsinki and its later amendments or comparable ethical standards.

Informed consent Written informed consent was obtained from all individual participants included in the study. All data are anonymised.

\section{References}

1. Sheetz KH, Claflin J, Dimick JB (2020) Trends in the adoption of robotic surgery for common surgical procedures. JAMA Netw Open 3:e1918911. https://doi.org/10.1001/jamanetworkopen .2019.18911

2. Weaver A, Steele S (2016) Robotics in colorectal surgery. F1000Research 5:2373. https://doi.org/10.12688/f1000resea rch.9389.1

3. Hewett PJ, Allardyce RA, Bagshaw PF et al (2008) Short-term outcomes of the australasian randomized clinical study comparing laparoscopic and conventional open surgical treatments for colon cancer: The ALCCaS Trial. Ann Surg 248:728-738. https://doi. org/10.1097/SLA.0b013e31818b7595

4. Hares L, Roberts P, Marshall K, Slack M (2019) Using end-user feedback to optimize the design of the versius surgical system, a new robot-assisted device for use in minimal access surgery. BMJ Surg Interv Health Technol 1:e000019. https://doi.org/10.1136/ bmjsit-2019-000019

5. Morton J, Hardwick RH, Tilney HS et al (2020) Preclinical evaluation of the versius surgical system, a new robot-assisted surgical device for use in minimal access general and colorectal procedures. Surg Endosc. https://doi.org/10.1007/s00464-020-07622 -4 (Published online)

6. St-Louis E, Iqbal S, Feldman LS et al (2015) Using the ageadjusted Charlson comorbidity index to predict outcomes in emergency general surgery. J Trauma Acute Care Surg 78:318-323. https://doi.org/10.1097/TA.0000000000000457

7. Dindo D, Demartines N, Clavien P-A (2004) Classification of surgical complications: a new proposal with evaluation in a cohort of 6336 patients and results of a survey. Ann Surg 240:205-213. https://doi.org/10.1097/01.sla.0000133083.54934.ae

8. van der Pas MHGM, Deijen CL, Abis GSA et al (2017) Conversions in laparoscopic surgery for rectal cancer. Surg Endosc 31:2263-2270. https://doi.org/10.1007/s00464-016-5228-8

9. Jayne D, Pigazzi A, Marshall H et al (2017) Effect of roboticassisted vs conventional laparoscopic surgery on risk of conversion to open laparotomy among patients undergoing resection for rectal cancer: The ROLARR Randomized Clinical Trial. JAMA 318:1569. https://doi.org/10.1001/jama.2017.7219

10. Waters PS, Cheung FP, Peacock O et al (2020) Successful patientoriented surgical outcomes in robotic vs laparoscopic right hemicolectomy for cancer-a systematic review. Colorectal Dis 22:488-499. https://doi.org/10.1111/codi.14822

Publisher's Note Springer Nature remains neutral with regard to jurisdictional claims in published maps and institutional affiliations. 